[13] T. H. Lee and J. C. Lin, "A fully distributed power control algorithm for cellular mobile systems," IEEE J. Sel. Areas Commun., vol. 14, no. 4, pp. 692-697, May 1996.

[14] R. D. Yates, "A framework for uplink power control in cellular radio systems," IEEE J. Sel. Areas Commun., vol. 13, no. 7, pp. 1341-1347, Sep. 1995.

[15] S. Haykin, Adaptive Filter Theory. Englewood Cliffs, NJ: Prentice-Hall, 1991.

\section{Iterative Irregular Sphere Detection in High-Rate Downlink SDMA Systems}

\author{
Chun-Yi Wei, Li Wang, and Lajos Hanzo
}

\begin{abstract}
Downlink spatial division multiple-access (DL-SDMA)assisted multiuser transmission (MUT) employing linear dispersion codes (LDCs) is proposed for space-time signaling, which attains a high diversity order and may support a high-rate data transmission. However, a potentially high complexity may be imposed by the signal detection when aiming to achieve optimum maximum-likelihood (ML) performance. Hence, a novel irregular sphere detection (IrSD) design is proposed to maintain an infinitesimally low bit error rate (BER) at the lowest possible iterative decoding complexity. Quantitatively, in the specific scenario, when six 4-quadratic-amplitude-modulated (QAM) data symbol streams were transmitted by the DL-SDMA system, the full-search-based ML detector had to evaluate all $4^{6}=4096$ possible decision candidates. By contrast, when searching through a fraction of this space, the proposed IrSD may reduce the complexity by three orders of magnitude, quantified in terms of the number of add-compare-select (ACS) arithmetic operations, namely, from the order of $10^{8}$ to about $10^{5}$ at $E_{b} / N_{0}=5 \mathrm{~dB}$, and still approach the ML performance.
\end{abstract}

Index Terms-Down-link, irregular code design, iterative detections, SDMA, sphere decoding, sphere detection.

\section{INTRODUCTION}

In this paper, we introduce a downlink multiuser system design for employment in high-rate wireless multimedia communication systems. By amalgamating linear dispersion codes (LDCs) [1] with the downlink spatial division multiple-access (DL-SDMA) system advocated in [2], a novel LDC-aided DL-SDMA scheme is proposed in this paper. The family of LDCs, which may be considered as a class of space-time block codes, exhibits high flexibility in terms of diversity versus multiplexing gain; hence, LDCs have attracted substantial research attention [3]-[5]. An LDC approaching the multiple-input-multipleoutput (MIMO) channel's capacity was proposed in [1], which was refined in [3] for the sake of satisfying different design criteria, such as minimizing the bit error ratio (BER) or the block error ratio [4]. Our LDC scheme was designed to maximize the sum rate of the DL-SDMA system, which may be achieved by adopting the rank-deficient scheme advocated in [2]. However, similar to the LDC-aided system designs of [3]-[5], a low-complexity detector is required for the proposed LDC-aided DL-SDMA system. As advocated in [2] and [6], sphere

Manuscript received July 10, 2008; revised November 21, 2008 and January 18, 2009. First published February 10, 2009; current version published August 14, 2009. The review of this paper was coordinated by Dr. D. Matolak.

C.-Y. Wei was with the School of Electronics and Computer Science, University of Southampton, Southampton SO17 1BJ, U.K. He is now with the National Taipei University, Taipei 104, Taiwan (e-mail:cywei@mail.ntpu.edu.tw).

L. Wang and L. Hanzo are with the School of Electronics and Computer Science, University of Southampton, Southampton SO17 1BJ, U.K. (e-mail: 1h@ecs.soton.ac.uk).

Digital Object Identifier 10.1109/TVT.2009.2015030 detection (SD) is capable of attaining a near-maximum-likelihood (ML) performance at a low complexity, and hence, it is particularly suitable for transmitting a high number of bits per symbols combined with many antennas. In contrast to other recent research efforts [6]-[8] attempting to reduce the complexity of each SD iteration, which might still result in a relatively high overall iterative decoding complexity, the main contribution of this paper is that we introduce the concept of irregular SD (IrSD), which was designed to reduce the overall iterative decoding complexity by taking into account both the per-iteration complexity and the number of iterations required. The proposed IrSD algorithm has the following attractive properties.

1) It is constructed by using our novel center-shifting (CS)-aided $K$-best sphere detector (KSD) scheme referred to as KSD-CS, which allows us to employ a reconfigurable number of SD candidates. The proposed KSD-CS is attractive because we can directly control its complexity by adjusting the number of SD candidates and achieve a near-ML performance at a fraction of the ML detector's complexity. Furthermore, the iterative decoding gain of our KSD-CS may substantially be improved, as compared with that of the original KSD [7], as we will illustrate in Section IV-B.

2) The design of the IrSD scheme may readily be accomplished with the aid of extrinsic information transfer (EXIT) charts [9]. The EXIT function of the IrSD-aided system and its convergence behavior can also readily be predicted and analyzed. In other words, the design of the IrSD arrangement can be simplified to an EXIT curve-fitting or matching problem, adopting a philosophy originally suggested for the design of near-capacity irregular convolutional codes (IRCCs) [10].

3) In contrast to IRCCs, the design of the IrSD requires finding the specific weighting coefficients for the various IrSD receiver components, which result in the lowest total complexity. Furthermore, this requires an "offline" optimization. Hence, its complexity does not contribute to the complexity of the real-time iterative decoding process.

The rest of this paper is structured as follows: First, we portray the LDC-aided DL-SDMA system model in Section II and detail our LDC design criteria in Section III. The design of the proposed IrSD is detailed in Section IV, whereas our performance results are provided in Section V. Finally, we conclude this paper in Section VI.

\section{SYSTEM MODEL}

The structure of the iterative-decoding-aided DL-SDMA systems using LDC-aided multiuser transmission (MUT) schemes is depicted in Fig. 1. Assume that our system uses $M$ transmit antennas at the base station (BS) for supporting $K$ mobile stations (MSs), while each MS employs $N_{k}$ receive antennas. We denote an $\left(N_{k} \times M\right)$-element channel matrix by $\boldsymbol{H}^{(k)}$, which is constituted by the sampled flat-fading channel impulse responses of each antenna element (AE) experienced by the $k$ th user. The elements $\left[\boldsymbol{H}^{(k)}\right]_{i, j}$, where we have $1 \leq i \leq$ $N_{k}$ and $1 \leq j \leq M$, are independent identically distributed complexvalued Gaussian random variables with a distribution $\mathcal{C N}(0,1){ }^{1}$

Motivated by the three-stage single-input-single-output turbo equalizer of [2], we improve the efficiency of the extrinsic information exchange of the conventional two-stage turbo receiver by intrinsically

\footnotetext{
${ }^{1}$ The notations employed are defined as follows: $[\boldsymbol{A}]_{i, j}$ indicates the $i$ th row and $j$ th column element of matrix $\boldsymbol{A} ;\left.b_{n}\right|_{n=1} ^{N}$ indicates the variables $b_{1}, b_{2}, \ldots, b_{N} ;[\cdot]^{\dagger}$ denotes the vector transpose; and diag $\mathbf{b}$ represents a diagonal matrix having the elements of the vector $\mathbf{b}$.
} 


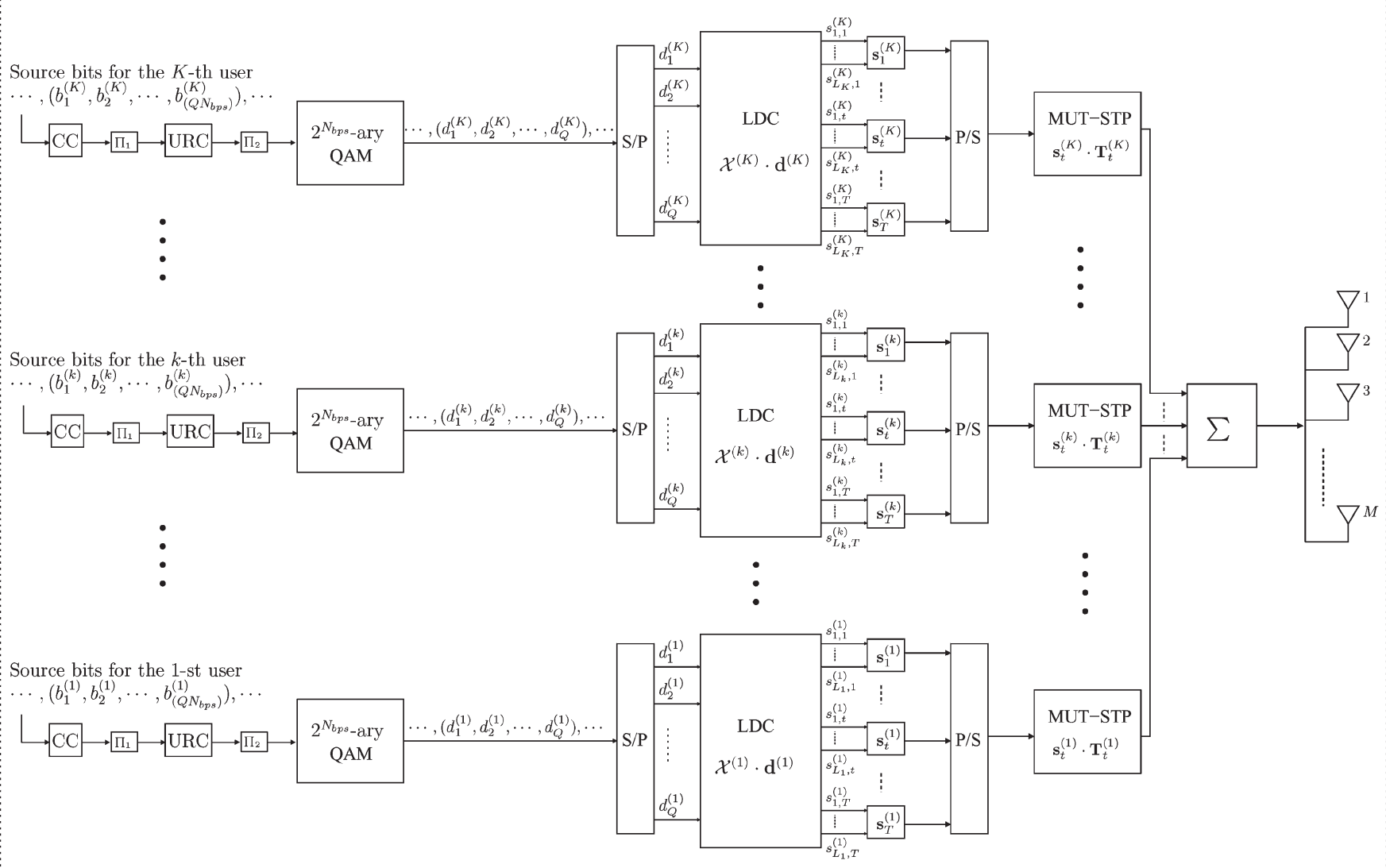

The transmitter of BS

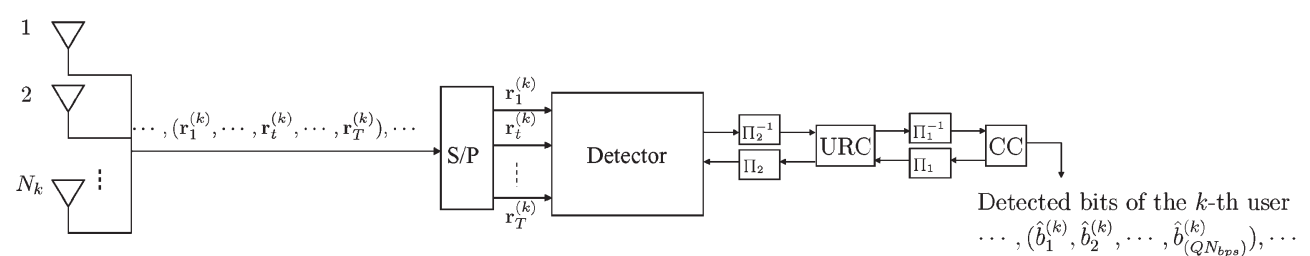

The receiver of the $k$-th MS

Fig. 1. LDC-aided MUT in the DL-SDMA system.

amalgamating the decoder of a convolutional code with that of a unityrate code (URC) having an infinite impulse response (IIR), both of which are embedded in a channel-coded spatial division multipleaccess (SDMA) transceiver, hence creating a powerful three-stage serially concatenated scheme. Explicitly, as illustrated in Fig. 1, the source bits $\left.b_{i}^{(k)}\right|_{i=1} ^{Q N_{b p s}}$ transmitted to the $k$ th user are encoded both by the channel encoder and the URC encoder [2], [11] before being modulated using an $\mathcal{M}$-ary quadratic-amplitude modulator (QAM), where we have $\mathcal{M}=2^{N_{b p s}}$, and $N_{b p s}$ denotes the number of bits per modulated symbol. The $Q$ QAM data symbols $\left.d_{n}^{(k)}\right|_{n=1} ^{Q}$, which constitute the data vector $\mathbf{d}^{(k)}$, are encoded into the so-called LDC symbol $\mathbf{S}^{(k)}$ by multiplying the data vector $\mathbf{d}^{(k)}$ with the designed linear dispersion matrix $\mathcal{X}^{(k)}$ [3], where the LDC symbol $\mathbf{S}^{(k)}$ is a hypervector constituted by $T$ column vectors, i.e., we have $\left.\mathbf{s}_{t}^{(k)}\right|_{t=1} ^{T}$. Each column vector $\mathbf{s}_{t}^{(k)}$ is constituted by the elements $\left.s_{j, t}^{(k)}\right|_{j=1} ^{L_{k}}$, where $L_{k}$ represents the number of independent signal streams contained in the DL-SDMA symbol stream $\mathbf{s}_{t}^{(k)}$. According to [2, eq. (6)], we define $L_{k}=M-\sum_{j=1, j \neq k}^{K} N_{j}$. Additionally, we define the so- called spatiotemporal preprocessor (STP) matrix $\boldsymbol{T}_{t}^{(k)} \in \mathbb{C}^{M \times L_{k}}$, which was designed to eliminate the multiuser interference (MUI) [12], as detailed in [2]. Briefly, $\boldsymbol{T}_{t}^{(k)}$ may be constructed based on the null-space of the matrix $\tilde{\boldsymbol{H}}_{t}^{(k)}$, where the elements of $\tilde{\boldsymbol{H}}_{t}^{(k)}$ are constituted by $\left.\boldsymbol{H}_{t}^{(i)}\right|_{i=1, i \neq k} ^{K}$ to prevent the user's signals from being transmitted to other users. Hence, the general received signal model of the $k$ th user is given by

$$
\boldsymbol{r}_{t}^{(k)}=\boldsymbol{H}_{t}^{(k)} \boldsymbol{T}_{t}^{(k)} \boldsymbol{s}_{t}^{(k)}+\sum_{i=1, i \neq k}^{K} \boldsymbol{H}_{t}^{(k)} \boldsymbol{T}_{t}^{(i)} \boldsymbol{s}_{t}^{(i)}+\boldsymbol{n}_{t}^{(k)}
$$

where the second term of (1) represents the MUI, which will entirely be eliminated when $\boldsymbol{T}_{t}^{(i)}$ is designed based on the perfect knowledge of $\left.\boldsymbol{H}_{t}^{(i)}\right|_{i=1} ^{K}$. Furthermore, $\mathbf{n}_{t}^{(k)}$ represents the additive white Gaussian noise (AWGN) vectors obeying the distribution $\mathcal{C N}\left(0, N_{o}\right)$ for each constituent element, where we have $N_{o}=2 \sigma_{n}^{2}$. 
Let $\mathbf{H}^{(k)}$ represent the observed spatial-temporal channel impulse response (ST-CIR) of the $k$ th user, which is assumed to be time variant over an interval of $T$ slots, i.e., we have $\mathbf{H}^{(k)}=\left[\boldsymbol{H}_{1}^{(k)}, \boldsymbol{H}_{2}^{(k)}, \ldots, \boldsymbol{H}_{t}^{(k)}, \ldots, \boldsymbol{H}_{T}^{(k)}\right]^{\dagger}$. Furthermore, let $\mathbf{T}^{(k)}=\left[\boldsymbol{T}_{1}^{(k)}, \boldsymbol{T}_{2}^{(k)}, \ldots, \boldsymbol{T}_{t}^{(k)}, \ldots, \boldsymbol{T}_{T}^{(k)}\right]^{\dagger}$. Therefore, we may express the effective channel matrix $\mathcal{H}^{(k)}$ experienced by the $k$ th user over the interval of $T$ slots, i.e., over the duration of an LDC symbol, as $\mathcal{H}^{(k)}=\operatorname{diag}\left(\mathbf{H}^{(k)}\right) \operatorname{diag}\left(\mathbf{T}^{(k)}\right)$. Hence, we denote $\mathbf{Y}^{(k)}$ as the $k$ th user received signal successively observed over an interval of $T$ slots, which is given by

$$
\mathbf{Y}^{(k)}=\mathcal{H}^{(k)} \mathcal{X}^{(k)} \mathbf{d}^{(k)}+\sum_{i=1, i \neq k}^{K} \mathcal{H}^{(k)} \mathcal{X}^{(i)} \mathbf{d}^{(i)}+\mathbf{N}^{(k)}
$$

where $\mathbf{N}^{(k)}$ is a $T$-element vector, which is constituted by the AWGN vectors $\left.\mathbf{n}_{t}^{(k)}\right|_{t=1} ^{T}$.

Fig. 1 also illustrates the structure of each MS receiver. First, the detector will detect the received signals of the $T$ slots. The extrinsic information exchange is carried out among the detector, the URC decoder, and the employed channel decoder.

\section{Design of the Linear Dispersion Matrix FOR HIGH-RATE SCENARIOS}

As mentioned in Section I, we aim to design an LDC for a highthroughput DL-SDMA multiuser system in this application. Therefore, by extending the LDC design of [3], we adopt the specific LDC that may maximize the sum rate of the DL-SDMA system. More specifically, we may design $\mathcal{X}^{(k)}$ of (2), where $k=1,2, \ldots, K$, to approach the attainable capacity as closely as possible, as advocated in [3]. For the full-rate scheme, i.e., $L_{k} T=Q_{k}, \mathcal{X}^{(k)}$, which assists the system in approaching its attainable capacity, may be constructed with the aid of orthogonal vectors [3] or, alternatively, by invoking the design concept of [5]. On the other hand, in the case of $Q_{k}>L_{k} T$, we have a rankdeficient scenario, leading to a potentially high-throughput transmission scheme. Assuming that discrete-input-continuous-output QPSK or $\mathcal{M}$-ary QAM signaling is used, we may conduct a numerical search to find that particular matrix $\mathcal{X}^{(k)}$ that maximizes the equivalent ergodic capacity for the $k$ th MS, as expressed in the following equation [3] $]^{2}$ :

$$
\begin{aligned}
\mathfrak{C}^{(k)}= & \max _{\operatorname{tr}\left(\mathcal{X}^{(k)}\left(\mathcal{X}^{(k)}\right)^{H}\right) \leq T}-\frac{1}{T} \\
& \times E\left\{\operatorname { l o g } _ { 2 } \left(\frac{1}{\mathcal{M}^{Q}\left(\pi N_{o}\right)^{N_{k}}} \sum_{\check{\mathbf{d}}(k) \in \mathcal{M}_{c}^{Q}} \exp \right.\right. \\
& \left.\left.\times\left(-\frac{1}{N_{o}}\left\|\mathbf{Y}^{(k)}-\mathcal{H}^{(k)} \mathcal{X}^{(k)} \check{\mathbf{d}}^{(k)}\right\|^{2}\right)\right)\right\} \\
& -\frac{1}{T} N_{k} \log _{2}\left(\pi e N_{o}\right)
\end{aligned}
$$

where the equivalent ergodic capacity $\mathfrak{C}^{(k)}$ of the system described in (2) is quantified by assuming a perfect ST-CIR at the transmitter. In other words, (3) may be used to describe the maximum sum rate of the DL-SDMA system, even if our knowledge of the ST-CIR is imperfect. Additionally, $\mathcal{M}_{c}$ denotes the set of $\mathcal{M}$ complex-valued constellation

\footnotetext{
${ }^{2}$ The LDC schemes of [1] and [3] were optimized based on maximizing the ergodic capacity, assuming that the continuous-input-continuous-output memoryless channel was used. However, our LDC scheme was optimized based on the ergodic capacity, assuming that discrete-input-continuous-output QPSK or $\mathcal{M}$-ary QAM signaling is used, as formulated in [8].
}

points of the employed modulation scheme, and $\mathcal{M}_{c}^{Q}$ is the total set of legitimate values hosted by the transmitted data symbol vector $\mathbf{d}^{(k)} . E\{\cdot\}$ here is taken with respect to different effective channel realizations $\mathcal{H}^{(k)}$ and to the QPSK or $\mathcal{M}$-ary QAM signals $\mathbf{d}^{(k)}$ and $\mathbf{N}^{(k)}$, respectively.

The higher the value of $Q$, the higher the downlink transmission rate, but naturally, this is achieved at a higher signal detection complexity. Therefore, efficient complexity reduction techniques are required, which was also the challenge of [1] and [3]-[5]. Against this background, we proposed a novel IrSD scheme to reduce the complexity imposed by the iterative joint detection and decoding process, which will be detailed in the following section.

\section{IRREgular Sphere Detection}

In practice, the required ST-CIR knowledge may be attained by using the estimation and prediction schemes of [13] and [14], which have to be further assisted by the ST-CIR feedback schemes of [15]. Naturally, accurate ST-CIR estimation/prediction has the potential of significantly reducing the residual MUI, which is represented by the second term of (2). However, owing to space limitations, here, we have to focus our attention on the proposed irregular SD by stipulating the simplifying assumption of having a perfect ST-CIR. The achievable complexity reduction remains valid in the presence of an imperfect ST-CIR, but the attainable performance is expected to erode to a degree. Additionally, for the sake of simplicity, we will remove the user index $k$, where this does not cause any confusion. Furthermore, by defining the effective channel matrix as $\mathcal{H}_{e}=\mathcal{H} \mathcal{X}$, we may write (2) as

$$
\mathbf{Y}=\mathcal{H} \mathcal{X} \mathbf{d}+\mathbf{N}=\mathcal{H}_{e} \mathbf{d}+\mathbf{N}
$$

\section{A. $k$-best Sphere Detector}

The ML solution of (2), which was further streamlined in (4), may equivalently be expressed as [6]

$$
\hat{\mathbf{d}}=\arg \min _{\breve{\mathbf{d}} \in \mathcal{M}_{c}^{Q}}\left\|\mathbf{U}\left(\breve{\mathbf{d}}-\hat{\mathbf{x}}_{c}\right)\right\|^{2}
$$

where $\mathbf{U}$ is a $(Q \times Q)$-element upper triangular matrix, and $\mathbf{U}^{H} \mathbf{U}=$ $\left(\mathcal{H}_{e}^{H} \mathcal{H}_{e}+2 \sigma_{n}^{2} \mathbf{I}\right)$. Again, $\mathcal{M}_{c}^{Q}$ is the set of all possible candidates of the $Q$-element transmitted data vector $\mathbf{d}$. Furthermore, $\hat{\mathbf{x}}_{c}$ is the transmitted data vector candidate that is considered to be the search center of the SD, which is usually the MMSE solution. Consequently, the objective function $\mathcal{J}$ associated with the candidate $\overrightarrow{\mathbf{d}}$ of the transmitted data vector, which will be evaluated during the SD's nearML search, is defined as [6]

$$
\begin{aligned}
\mathcal{J}(\breve{\mathbf{d}}) & =\left\|\mathbf{U}\left(\breve{\mathbf{d}}-\hat{\mathbf{x}}_{c}\right)\right\|^{2}=\left(\breve{\mathbf{d}}-\hat{\mathbf{x}}_{c}\right) \mathbf{U}^{H} \mathbf{U}\left(\breve{\mathbf{d}}-\hat{\mathbf{x}}_{c}\right) \\
& =\sum_{i=1}^{Q}\left|\sum_{j=i}^{Q} u_{i j}\left(\breve{d}_{j}-\hat{x}_{c, j}\right)\right|^{2}=\sum_{i=1}^{Q} \phi_{i}\left(\breve{\mathbf{d}}_{i}\right)
\end{aligned}
$$

where we have $\breve{\mathbf{d}}_{i}=\left(\breve{d}_{i}, \ldots, \breve{d}_{Q}\right)$. Furthermore, we define the partial Euclidean distance (PED) as $\mathcal{J}_{i}\left(\breve{\mathbf{d}}_{i}\right)=\mathcal{J}_{i+1}\left(\breve{\mathbf{d}}_{i+1}\right)+\phi_{i}\left(\breve{\mathbf{d}}_{i}\right)$, where we have $i=1, \ldots, Q$.

In general, the SD's near-ML search may be depicted as a tree search process [6]. In the class of KSDs [7], at each layer of the search, i.e., when emerging from the layer $i=1$ to the layer $i=Q$, the KSD only retains a reduced and fixed number of candidates $N_{\text {cand }}$, which have the smallest PEDs. For example, assuming that we have $N_{\text {cand }}=4$, at 
TABLE I

SYSTEM PARAMETERS

\begin{tabular}{|l|r|}
\hline Channel model & uncorrelated flat-fading MIMO channel \\
\hline $\begin{array}{l}\text { Channel Coder } \\
\text { Interleaver length }\end{array}$ & rate-0.5 RSC [5,7] \\
$10^{5}$ bits \\
\hline URC Coder & rate-1 Conv. Coder [18] \\
\hline Modulation & $4 Q A M$ \\
\hline Number of users & $K=3$ \\
\hline Total number of transmit antennas & $M=7$ \\
\hline Number of receive antennas of the $k$-th user & $N_{k}=2$, for $k=1,2,3$. \\
\hline Total number of receive antennas & 6 \\
\hline Dimension in the spatial domain & $L_{k}=3$, for $k=1,2,3$. \\
\hline Number of independent data streams transmitted for the $k$-th user in one LDC symbol & $Q_{k}=6$, for $k=1,2,3$. \\
\hline The interval of one LDC symbol & $T=2$ \\
\hline
\end{tabular}

the bottom of the search tree, there will be only $N_{\text {cand }}=4$ candidates left, as compared with the $256\left(4^{4}\right)$ candidates of the full-search-based ML detector.

By defining $\mathcal{D}_{\mathfrak{K}} \subset \mathcal{M}_{c}^{Q}$ as the reduced hypothesis set containing $N_{\text {cand }}=\mathfrak{K}$ candidates only, the desired a posteriori log-likelihood ratio (LLR) of the $i$ th bit of the $n$th symbol element of the transmitted data vector $\mathbf{d}$ formulated in (4) can be expressed as [16]

$$
\begin{aligned}
\mathcal{L}_{\text {apt }}^{\text {det }}\left(\mathbf{d}_{i, n}\right)= & \min _{\check{\mathbf{d}} \in \mathcal{D}_{\mathfrak{K},(i, n)}^{0}}\left\{-\log p(\check{\mathbf{d}})+\frac{\left\|\mathbf{Y}-\mathcal{H}_{e} \check{\mathbf{d}}\right\|^{2}}{2 \sigma_{n}^{2}}\right\} \\
& -\min _{\check{\mathbf{d}} \in \mathcal{D}_{\mathfrak{K},(i, n)}^{1}}\left\{-\log p(\check{\mathbf{d}})+\frac{\left\|\mathbf{Y}-\mathcal{H}_{e} \check{\mathbf{d}}\right\|^{2}}{2 \sigma_{n}^{2}}\right\}
\end{aligned}
$$

where the hypothesis set $\mathcal{D}_{\mathfrak{K},(i, n)}^{b}$ contains the candidates that have their $i$ th bit of the $n$th symbol element equal to the binary bit $b=$ $\{0,1\}$. In addition, $p(\mathbf{d})$ is the a priori soft information of the given hypothetical data vector being considered.

\section{B. KSD Using CS}

The system parameters are summarized in Table I. For the sake of maintaining a moderate complexity imposed by the KSD, a relatively small candidate list size $N_{\text {cand }}$ has to be employed. However, when the KSD is operating in a high-rate system, such as, for example, the downlink of the SDMA system depicted in Fig. 1, the value of $N_{\text {cand }}$ has to be accordingly increased to maintain a desirable performance. Otherwise, there might not be any iterative gain achieved upon increasing the extrinsic information provided for the KSD, as demonstrated by our EXIT analysis in Fig. 2, where we observe that the KSD's EXIT curve decayed upon increasing the a priori information owing to the flawed information exchange between the inner and outer decoders, which was caused by the employment of an insufficiently large candidate list size $N_{\text {cand }}$.

Therefore, to still maintain a moderate complexity imposed by the $\mathrm{KSD}$, while achieving a desirable performance in high-rate systems, we found that in the context of the iterative joint detection and decoding schemes, this search center $\hat{\mathbf{x}}_{c}$ in (5) can be updated at each iteration based on the a priori information input, which is expected to become more reliable upon using more iterations. Hence, by referring to this process as CS, we introduce the CS-aided KSD concept. Additionally, by employing the a priori information-aided MMSEbased center calculation [17], a new center $\hat{\mathbf{x}}_{c}$ will be generated at each iteration according to the input $a$ priori information updated by using the extrinsic information output of the channel decoder. Based on the updated center $\hat{\mathbf{x}}_{c}$, the KSD updates the set $\mathcal{D}_{\mathfrak{K}}$ constituted by the $N_{\text {cand }}$ number of SD candidates having the smallest PED.

Our EXIT analysis in Fig. 2 demonstrated that with the aid of CS, the KSD scheme exhibits a substantial iterative gain for both $N_{\text {cand }}=$

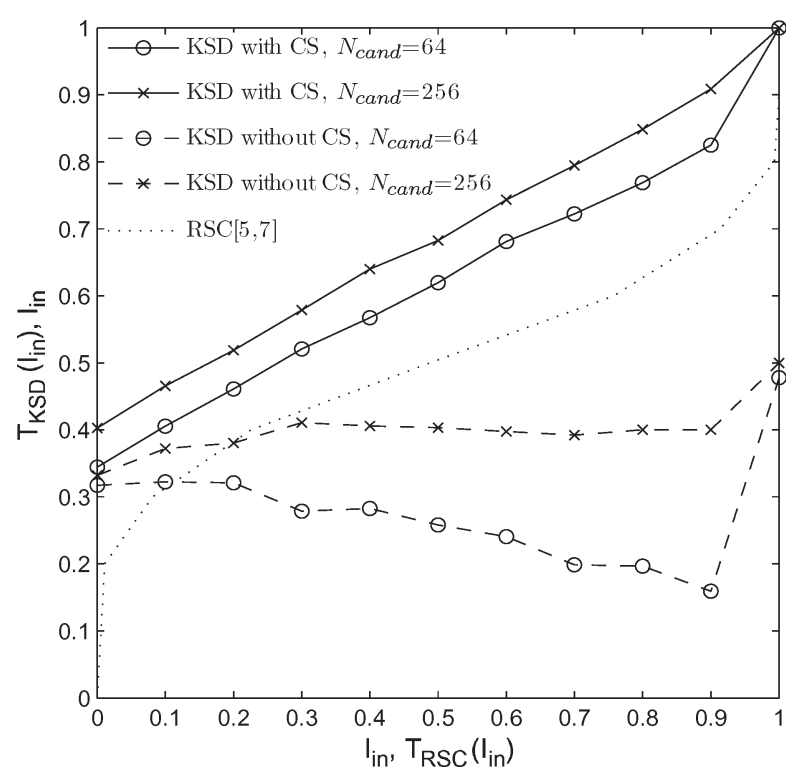

Fig. 2. EXIT chart analysis of the iterative KSD using the a priori information-aided MMSE CS scheme. A rate-0.5 RSC channel codec, having the octal generator polynomials of $G=[5,7]$, was employed by the system. The systems were operated at $E_{b} / N_{0}=5 \mathrm{~dB}$. The system parameters of Table I were used. Additionally, $T_{\mathrm{RSC}}\left(I_{\mathrm{in}}\right)$ represents the EXIT function of the employed RSC decoder, whereas $T_{\mathrm{KSD}}\left(I_{\mathrm{in}}\right)$ represents the EXIT function of KSD detectors.

64 and $N_{\text {cand }}=256 \mathrm{KSD}$ candidates. Furthermore, since the employed URC has an IIR due to its recursive coding structure, the EXIT chart curve is capable of reaching the highest point $(1,1)$, provided that the interleaver length is sufficiently large. On the other hand, since the URC decoder employs the maximum a posteriori probability decoding scheme, the extrinsic probability computed at the output of the URC decoder contains the same amount of information as the sequence at the input of the URC decoder. In other words, the area under the inner EXIT curve remains unchanged, regardless of the employment of the URC [18]. Hence, a higher endpoint of the EXIT curve leads to a lower starting point, implying a steeper slope for the EXIT curve.

\section{C. $\operatorname{IrSD}$}

In this section, we introduce the framework of IrSD by appropriately amalgamating a number of CS-aided KSDs, each using a different value of $N_{\text {cand }}$, although this may simply be interpreted as using a single KSD-CS and a variable value of $N_{\text {cand }}$. The CS-aided KSD using different values of $N_{\text {cand }}$ has a different decoding performance and a different detection complexity. Hence, by invoking appropriately 
amalgamated KSD-CS associated with different values of $N_{\text {cand }}$, the IrSD scheme is designed to reduce the overall complexity, which takes into account both the complexity per iterative detection stage and the number of iterations required, with each constituent KSD-CS processing the required fraction of the received bits.

More specifically, the philosophy of the IrSD technique is that of utilizing $V$ KSDs employing different values of $N_{\text {cand }}$ for detecting predetermined segments of the transmitted signals, instead of using a fixed value of $N_{\text {cand }}$, as justified later in this section. Based on the appropriately optimized fractions of the received signal, each KSD-CS generates its soft-bit estimates of the appropriate fractions of the transmitted signals. More specifically, for a transmission block containing $L$ encoded bits and assuming that $N_{b p s}$ bits per symbol are transmitted by the modulator, we have $L / N_{b p s}$ symbols per transmission block, where each transmission block is mapped to a separate time slot. At the receiver, we invoke the proposed IrSD scheme, which is constituted by $V$ component KSD-CS employing different values of $N_{\text {cand }}$. Let $C_{v}$ be the complexity of the $v$ th component detector of the IrSD scheme. Then, the weighting coefficient $\alpha_{v}, v=1, \ldots, V$ of the KSD-CS has to satisfy

$$
1=\sum_{v=1}^{V} \alpha_{v}, \quad C_{\mathrm{gd}}=\sum_{v=1}^{V} \alpha_{v} C_{v}, \quad \text { and } \quad \alpha_{v} \in[0,1] \quad \forall v
$$

where $C_{\mathrm{gd}}$ is the average complexity of the IrSD scheme. ${ }^{3}$

For each iterative stage, the computational complexity of the $v$ th KSD-CS associated with the given $N_{\text {cand }}=\mathfrak{K}$ can be quantified as

$$
C_{v}=Q \cdot N_{b p s} \cdot \mathbf{\Phi}(\mathfrak{K})
$$

whereas before, $Q$ is the number of elements in the transmitted signal vector $\mathbf{d}$, and $\Phi(\mathfrak{K})$ is the computational complexity of evaluating each bit's LLR in $\mathbf{d}$ formulated in (7) and associated with the reduced hypothesis set $\mathcal{D}_{\mathfrak{K}}$.

Furthermore, each constituent detector generates $\alpha_{v} L$ soft bits for a transmission block containing $L$ bits. In contrast to the irregular scheme of [10], which integrated a number of different-rate recursive systematic convolutional (RSC) decoders, the IrSD design philosophy is to superimpose the EXIT curves of the individual component detectors-i.e., "inner decoders" rather than outer decoders-after appropriately weighting them by the optimum value of $\alpha_{v}$, which facilitates the matching of the detector's EXIT curve with that of the outer channel decoder. To elaborate a little further, the EXIT function $T_{\mathrm{gd}}\left(I_{\mathrm{in}}\right)$, which characterizes the detector that is also synonymously referred to as the "inner decoder" of the IrSD-aided system, is given by

$$
T_{\mathrm{gd}}\left(I_{\mathrm{in}}\right)=\sum_{v=1}^{V} \alpha_{v} T_{v}\left(I_{\mathrm{in}}\right)
$$

where $T_{v}\left(I_{\text {in }}\right)$ is the EXIT function of the $v$ th component KSD-CS invoked by the IrSD scheme.

In summary, using the weighting coefficient vector $\boldsymbol{\alpha}$ formed by the weighting coefficients $\alpha_{v}$ for $v=1,2, \ldots, V$ satisfying (8) creates an IrSD having a given average computational complexity of $C_{\mathrm{gd}}$. Given the weighting coefficient vector $\boldsymbol{\alpha}$, the EXIT function of $T_{\mathrm{gd}}\left(I_{\mathrm{in}}\right)$ characterizing the inner decoder's EXIT curve can be generated based on (10). Therefore, we can design the superimposed EXIT function $T_{\mathrm{gd}}\left(I_{\mathrm{in}}\right)$ of the IrSD-aided system by optimizing the weighting coefficient vector $\boldsymbol{\alpha}$ for the sake of minimizing the open EXIT tunnel area

\footnotetext{
${ }^{3}$ The subscript "gd" indicates generic detection, potentially using arbitrary detectors. However, in this paper, we use the KSD-CS in conjunction with different values of $N_{\text {cand }}$ employed as the component detectors.
}

and, hence, facilitating near-capacity operation, while maintaining an infinitesimally low BER.

\section{Optimization of the IrSD}

The weighting coefficient vector $\boldsymbol{\alpha} \in \mathbb{R}^{V}$ has to satisfy (8) and (10). Let $\mathcal{F}$ be a set containing all the candidate solutions $\boldsymbol{\alpha}$. We may find the weighting coefficient vector $\alpha \in \mathcal{F}$ by conducting a search similar to that suggested in [10]. By defining a $(P \times V)$-element matrix $\boldsymbol{A}$, which is constituted by $V$ number of EXIT functions $T_{j}\left(I_{\text {in }}\right), j=1$, $\ldots, V$, and a vector $\mathbf{b}=T_{\mathrm{cc}}^{-1}\left(I_{\text {in }}\right)$, for $I_{\text {in }} \in\left\{i_{1}, i_{2}, \ldots, i_{P}\right\}$, we have

$$
\begin{aligned}
& \mathbf{A}= {\left[\begin{array}{cccc}
T_{1}\left(i_{1}\right) & T_{2}\left(i_{1}\right) & \ldots & T_{V}\left(i_{1}\right) \\
T_{1}\left(i_{2}\right) & T_{2}\left(i_{2}\right) & \ldots & T_{V}\left(i_{2}\right) \\
\vdots & \vdots & \ldots & \vdots \\
T_{1}\left(i_{P}\right) & T_{2}\left(i_{P}\right) & \ldots & T_{V}\left(i_{P}\right)
\end{array}\right] } \\
& \mathbf{b}=\left[\begin{array}{c}
T_{\mathrm{cc}, 1}^{-1}\left(i_{1}\right) \\
T_{\mathrm{cc}, 1}^{-1}\left(i_{2}\right) \\
\vdots \\
T_{\mathrm{cc}, 1}^{-1}\left(i_{P}\right)
\end{array}\right] .
\end{aligned}
$$

Our objective function $\Omega(\boldsymbol{\alpha})$ may be defined as

$$
\Omega(\boldsymbol{\alpha})=\|\mathbf{A} \boldsymbol{\alpha}-\mathbf{b}\|^{2}
$$

which represents the area between the inner and outer channel decoders' EXIT curve, where the former is constituted by the combined IrSD and URC decoder. Naturally, all elements of $e$, where we have $\boldsymbol{e}=(\mathbf{A} \boldsymbol{\alpha}-\mathbf{b})$, have to be larger than zero, since they physically represent the area between the outer and inner decoders' EXIT curve. We define a subset $\mathcal{A} \subset \mathcal{F}$ containing all weighting coefficient vectors $\alpha$ meeting this constraint. Second, for a specific fixed computational complexity $C_{\mathrm{gd}}$, we opt for that particular $\boldsymbol{\alpha}$ value, which is associated with a higher area $\Omega(\boldsymbol{\alpha})$ between the outer EXIT curve $T_{\mathrm{cc}}^{-1}\left(I_{\mathrm{in}}\right)$ and the inner EXIT curve $T_{\text {gd }}\left(I_{\text {in }}\right)$, which implies requiring a low number of decoding iterations. Therefore, we assume that the optimal weighting coefficient vector $\boldsymbol{\alpha}_{\text {opt }}$ can be obtained by finding the $\boldsymbol{\alpha}$ value satisfying

$$
\boldsymbol{\alpha}_{\mathrm{opt}}=\max _{\boldsymbol{\alpha} \in \mathcal{A}} \Omega(\boldsymbol{\alpha})
$$

\section{E. IrSD Design and Analysis Using EXIT Charts}

In this section, we will demonstrate how EXIT charts may be used to assist us in the design of the IrSD and in the analysis of the systems' iterative decoding performance. Let us first define the IrSD's target complexity ratio $r_{\mathrm{gd}}$ with respect to the full candidate list searched by the KSD, i.e., by the ML, which is expressed as

$$
r_{\mathrm{gd}}=C_{\mathrm{gd}} / C_{\mathrm{ml}}
$$

where again, $C_{\mathrm{gd}}$ is the per-iteration computational complexity of the IrSD.

Furthermore, the total complexity $C_{\mathrm{gd} \text {,iter }}$ of the IrSD embedded into the iterative decoding process, which is defined as the product of the per-iteration complexity and the number of iterations required for approaching an infinitesimally low BER, is given by

$$
C_{\mathrm{gd}, \text { iter }}=C_{\mathrm{gd}} \times I_{\mathrm{gd}}
$$

where $I_{\mathrm{gd}}$ is the number of IrSD iterations required to achieve convergence to the $\left(I_{A}, I_{E}\right)=(1,1)$ point in the EXIT chart.

In Fig. 3, we illustrate the EXIT curves of the LDC-MUT-aided DLSDMA system employing the KSD-CS in conjunction with different values of $N_{\text {cand }}$. Observe that the systems employing the KSD-CS 


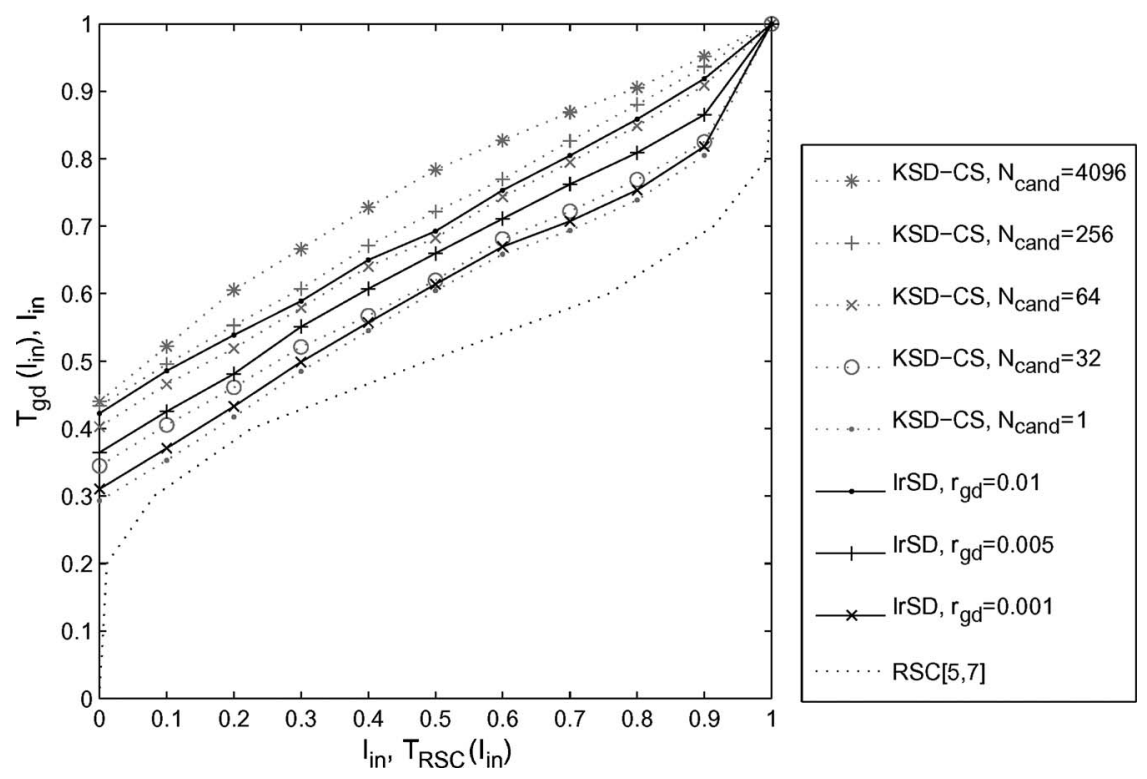

Fig. 3. EXIT curves of the LDC-MUT-aided DL-SDMA system employing the IrSD associated with $r_{\mathrm{gd}}=0.01,0.005$, and 0.001 and the corresponding weighting vectors $\boldsymbol{\alpha}=[0.37,0.03,0.6,0,0],[0.62,0.18,0.2,0,0]$, and $[0.93,0.07,0,0,0]$, respectively. The systems were operated at $E_{b} / N_{0}=5 \mathrm{~dB}$. The system employing the KSD-CS detector associated with different values of $N_{\text {cand }}$ is provided as a benchmark. The channel model was a flat-fading MIMO channel, and the system parameters of Table I were used. Additionally, $T_{\mathrm{gd}}\left(I_{\mathrm{in}}\right)$ represents the EXIT function of the $\operatorname{IrSD}$, whereas $T_{\mathrm{RSC}}\left(I_{\mathrm{in}}\right)$ represents the EXIT function of the employed RSC decoder.

in conjunction with different values of $N_{\text {cand }}$ have different inner EXIT curves. According to (9), the higher the value of $N_{\text {cand }}$, the higher the complexity at each detection stage. On the other hand, the wider EXIT tunnel of the system using higher values of $N_{\text {cand }}$ implies necessitating fewer iterative steps for the iterative process to converge. Hence, striking an attractive compromise between the value of $N_{\text {cand }}$ and the number of iterations has the potential of reducing the overall complexity. As an example, in Fig. 3, we characterize the IrSD associated with $r_{\mathrm{gd}}=0.01,0.005$, and 0.001 using the weighting vectors $\boldsymbol{\alpha}=[0.37,0.03,0.6,0,0],[0.62,0.18,0.2,0,0]$, and [0.93, 0.07, 0, $0,0]$, respectively, where the elements of $\boldsymbol{\alpha}$, from the first element to the last element, represent the KSD-CS using $N_{\text {cand }}=1,32,64$, 256 , and 4096, in this order. For example, for the IrSD associated with $r_{\mathrm{gd}}=0.005$ and $\boldsymbol{\alpha}=[0.62,0.18,0.2,0,0]$, the constituent KSD-CS using $N_{\text {cand }}=1$ has a $62 \%$ duty cycle, the KSD-CS using $N_{\text {cand }}=$ 32 has an $18 \%$ duty cycle, whereas the KSD-CS using $N_{\text {cand }}=64$ has a $20 \%$ duty cycle. Observe in Fig. 3 that when the IrSD is associated with smaller $r_{\text {gd }}$ values, the EXIT curves of the IrSD are moved closer to the EXIT curve of the employed RSC channel decoder.

In Fig. 4, we recorded the computational complexity $C_{\mathrm{gd} \text {,iter }}$ of the IrSD associated with different values of $r_{\mathrm{gd}}$. Additionally, the computational complexity of $C_{\mathrm{gd} \text {, iter }}$ may further be quantified in terms of the number of add-compare-select (ACS) operations, when considering the joint complexity of the IrSD detector, the URC decoder, and the employed RSC [5], [7] channel codec. Observe in Fig. 4 that the complexity $C_{\mathrm{gd} \text {,iter }}$ of the IrSD is reduced when we use a smaller $r_{\text {gd }}$ value. Therefore, we may search for the IrSD associated with $r_{\mathrm{gd}}$ having the lowest complexity, until the IrSD associated with $r_{\mathrm{gd}}$ exhibits no open EXIT tunnel, as shown in Fig. 3. Additionally, the total computational complexities associated with the KSD-CS using a fixed number of $N_{\text {cand }}=1,32,64,256$, and 4096 were provided in Fig. 4 as further benchmarks.

\section{Performance Results}

In Fig. 5(a), we recorded the imposed computational complexity, which is quantified by using the number of ACS operations in the iterative DL-SDMA system designed to reach a target BER of $10^{-5}$.

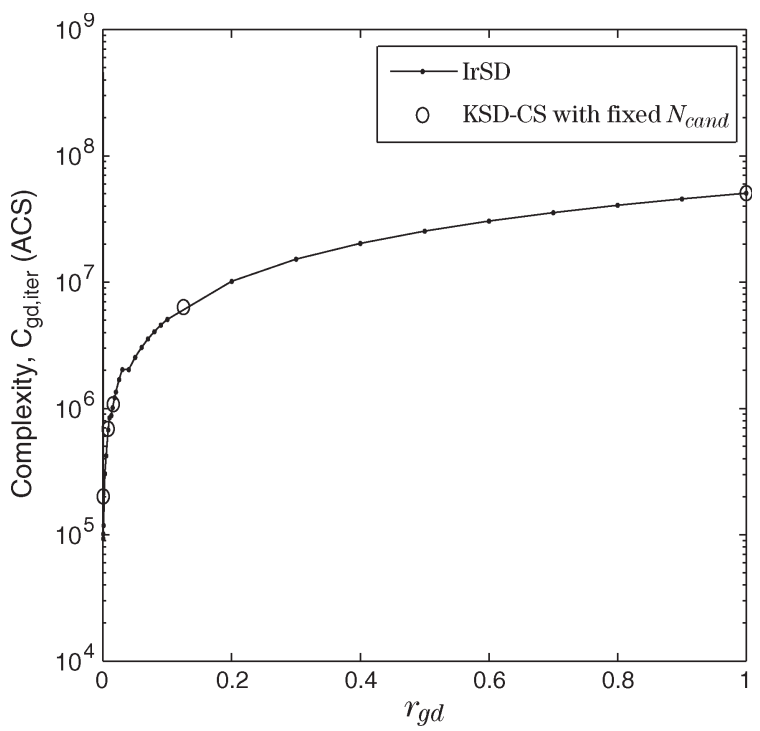

Fig. 4. Total complexity of the iterative IrSD decoding process. The systems were operated at $E_{b} / N_{0}=5 \mathrm{~dB}$. The system parameters of Table I were used.

Observe in Fig. 5(a) that the IrSD-aided iterative DL-SDMA systems have a lower computational complexity than the other KSD-CS detection-aided systems employing a fixed value of $N_{\text {cand }}$, where the IrSD employs $N_{\text {cand }}=1,64,128,256$, and 4096 . For example, at $E_{b} / N_{0}=5 \mathrm{~dB}$, the IrSD-aided DL-SDMA system has the lowest iterative decoding complexity, when we have $r_{\mathrm{gd}}=0.001$, and the corresponding weighting coefficient vector is $\boldsymbol{\alpha}=[0.93,0.07,0,0,0]$. Moreover, when the system is operated at $E_{b} / N_{0}=3 \mathrm{~dB}$, the KSD-CS(4096) detector may still maintain an open EXIT tunnel between its EXIT curve and the outer channel coder's EXIT curve. However, the EXIT curves of the other inner benchmark detectors, such as the KSD-CS(1), KSD-CS(32), KSD-CS(64), and KSD-CS(256) schemes, may intersect the EXIT curve of the outer channel encoder at $E_{b} / N_{0}=3 \mathrm{~dB}$. This resulted in the "infinite computational complexity" of the iterative decoding process imposed by the benchmarker 


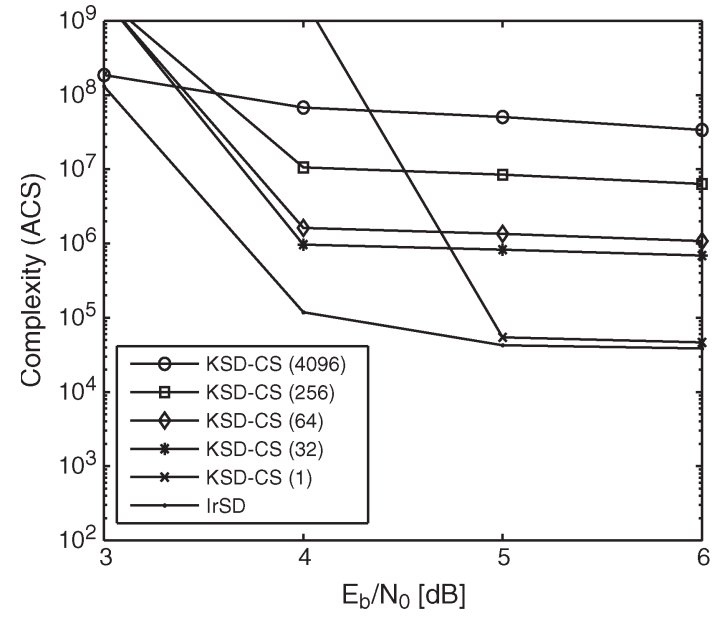

(a)

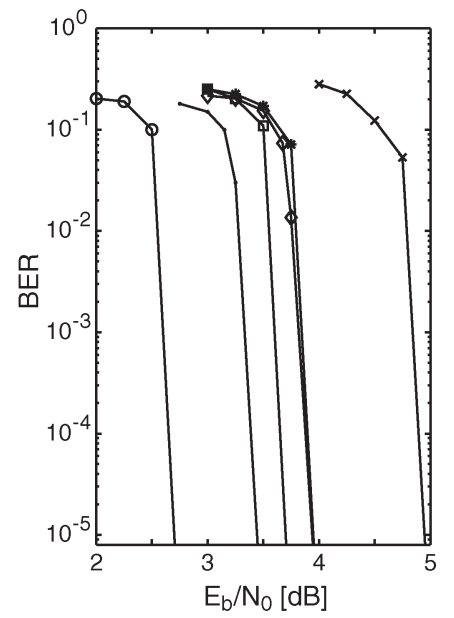

(b)

Fig. 5. Comparison of the (a) computational complexity per detected bit and (b) BER of the iterative-decoding-aided DL-SDMA system employing different detectors. The channel model was a flat-fading MIMO channel, and the parameters of Table I were used. The weighting coefficient vector $\boldsymbol{\alpha}$ of the IrSD employed at the $E_{b} / N_{0}$ values of $3,4,5$, and $6 \mathrm{~dB}$ were $[0,0,0,0.34,0.66]$, [0.37, 0.03, 0.6, 0, 0], [0.93, 0.07, 0, 0, 0], and [0.995, 0.005, 0, 0, 0], respectively.

detectors, because they would require an infinite number of iterations to achieve a target BER of $10^{-5}$. In Fig. 5(a), the crossover between the curve portraying the computational complexity imposed by the KSD-CS(4096) detector and those of the other benchmark detectors indicated this phenomenon.

Fig. 5(b) shows that the IrSD detector has only a modest $E_{b} / N_{0}$ degradation of about $1 \mathrm{~dB}$ compared with the KSD-CS benchmark associated with $N_{\text {cand }}=4096$. The KSD-CS detector using $N_{\text {cand }}=$ 4096 conducted a full search over the set of $\mathcal{M}^{Q}=4^{6}$ candidates, i.e., by taking into account all the possible candidates. By contrast, the KSD-CS detector using $N_{\text {cand }}=1$ represents the MMSE detector. Therefore, the proposed IrSD provides a flexible detection framework. By switching the value of $N_{\text {cand }}$ of KSD-CS detector according to the appropriately designed weighting coefficient vector $\boldsymbol{\alpha}$, the system may be expected to operate at the lowest possible iterative decoding complexity.

\section{CONCLUSion}

In this paper, we have demonstrated the LDC-aided DL-SDMA system, which had a high-diversity order and supported a high-rate data transmission. Moreover, we have proposed the IrSD algorithm, which is designed to reduce the complexity of iterative decoding in the LDCaided DL-SDMA system. By appropriately designing the weighting coefficient vector $\boldsymbol{\alpha}$, the IrSD-aided system may potentially provide the lowest possible iterative decoding complexity. Quantitatively, Fig. 5 suggests that the IrSD-aided iterative DL-SDMA system associated with $\boldsymbol{\alpha}=[0.93,0.07,0,0,0]$ may reduce the complexity by three orders of magnitude at $E_{b} / N_{0}=5 \mathrm{~dB}$, when compared with the KSD-CS benchmark detector associated with $N_{\text {cand }}=4096$.

\section{REFERENCES}

[1] B. Hassibi and B. Hochwald, "High-rate codes that are linear in space and time," IEEE Trans. Inf. Theory, vol. 48, no. 7, pp. 1804-1824, Jul. 2002.

[2] C.-Y. Wei, J. Akhtman, S. X. Ng, and L. Hanzo, "Iterative near-maximumlikelihood detection in rank-deficient downlink SDMA systems," IEEE Trans. Veh. Technol., vol. 57, no. 1, pp. 653-657, Jan. 2008.

[3] R. J. Heath and A. Paulraj, "Linear dispersion codes for MIMO systems based on frame theory," IEEE Trans. Signal Process., vol. 50, no. 10, pp. 2429-2441, Oct. 2002.

[4] X. Wang, V. Krishnamurthy, and J. Wang, "Stochastic gradient algorithms for design of minimum error-rate linear dispersion codes in MIMO wire- less systems," IEEE Trans. Signal Process., vol. 54, no. 4, pp. 1242-1255, Apr. 2006.

[5] F. Oggier, G. Rekaya, J. C. Belfiore, and E. Viterbo, "Perfect space-time block codes," IEEE Trans. Inf. Theory, vol. 52, no. 9, pp. 3885-3902, Sep. 2006.

[6] J. Akhtman, A. Wolfgang, S. Chen, and L. Hanzo, "An optimizedhierarchy-aided approximate log-MAP detector for MIMO systems," IEEE Trans. Wireless Commun., vol. 6, no. 5, pp. 1900-1909, May 2007.

[7] K.-W. Wong, C.-Y. Tsui, R. Cheng, and W.-H. Mow, "A VLSI architecture of a $K$-best lattice decoding algorithm for MIMO channels," in Proc. IEEE Int. Symp. Circuits Syst., 2002, vol. 3, pp. 273-276.

[8] B. Hochwald and S. ten Brink, "Achieving near-capacity on a multipleantenna channel," IEEE Trans. Commun., vol. 51, no. 3, pp. 389-399, Mar. 2003.

[9] S. ten Brink, "Designing iterative decoding schemes with the extrinsic information transfer chart," AË̈ Int. J. Electron. Commun., vol. 54, no. 6, pp. 389-398, 2000.

[10] M. Tüchler and J. Hagenauer, "EXIT charts of irregular codes," in Proc. Conf. Inf. Sci. Syst., Princeton, NJ, Mar. 2002, pp. 748-753.

[11] D. Divsalar, S. Dolinar, and F. Pollara, "Serial turbo trellis coded modulation with rate-1 inner code," in Proc. IEEE Global Telecommun. Conf., 2000, vol. 2, pp. 777-782.

[12] L.-U. Choi and R. Murch, "A transmit preprocessing technique for multiuser MIMO systems using a decomposition approach," IEEE Trans. Wireless Commun., vol. 3, no. 1, pp. 20-24, Jan. 2004.

[13] J. Akhtman and L. Hanzo, "Advanced channel estimation for MIMO-OFDM in realistic channel conditions," in Proc. IEEE Int. Conf. Commun., Jun. 2007, pp. 2528-2533.

[14] J. Akhtman and L. Hanzo, "Channel impulse response tap prediction for time-varying wireless channels," IEEE Trans. Veh. Technol., vol. 56, no. 5, pp. 2767-2769, Sep. 2007.

[15] D. J. Love, R. W. J. Heath, W. Santipach, and M. L. Honig, "What is the value of limited feedback for MIMO channels?" IEEE Commun. Mag., vol. 42, no. 10, pp. 54-59, Oct. 2004.

[16] L. Hanzo and T. Keller, OFDM and MC-CDMA: A Primer. New York: Wiley, 2006.

[17] M. Tuchler, A. C. Singer, and R. Koetter, "Minimum mean squared error equalization using a priori information," IEEE Trans. Signal Process., vol. 50, no. 3, pp. 673-683, Mar. 2002.

[18] L. Hanzo, T. Liew, and B. Yeap, Turbo Coding, Turbo Equalisation and Space-Time Coding. New York: Wiley, 2002. 